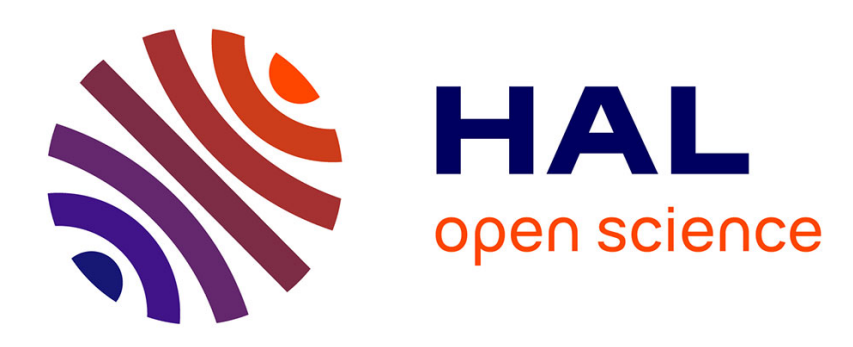

\title{
Evolution of cluster free energy during precipitation in concentrated binary alloys
}

\author{
Joel Lepinoux
}

\section{To cite this version:}

Joel Lepinoux. Evolution of cluster free energy during precipitation in concentrated binary alloys. Philosophical Magazine Letters, 2020, 100 (8), pp.402-411. 10.1080/09500839.2020.1777340 . hal02922107

\section{HAL Id: hal-02922107 \\ https://hal.science/hal-02922107}

Submitted on 15 Sep 2020

HAL is a multi-disciplinary open access archive for the deposit and dissemination of scientific research documents, whether they are published or not. The documents may come from teaching and research institutions in France or abroad, or from public or private research centers.
L'archive ouverte pluridisciplinaire HAL, est destinée au dépôt et à la diffusion de documents scientifiques de niveau recherche, publiés ou non, émanant des établissements d'enseignement et de recherche français ou étrangers, des laboratoires publics ou privés. 


\title{
Evolution of cluster free-energy during precipitation in concentrated binary alloys
}

\author{
J. Lépinoux \\ Univ. Grenoble Alpes, CNRS, Grenoble INP, SIMaP, F-38000 Grenoble, France \\ Corresponding author: joel.lepinoux@grenoble-inp.fr
}

\begin{abstract}
Considering ordered and coherent $\mathrm{Al}_{3} \mathrm{Li}$ clusters embedded in concentrated $\mathrm{AlLi}$ alloys as a model case, precipitation is investigated using Atomistic Kinematic Monte Carlo (AKMC) simulations. The free energy of clusters is extracted from simulations as a function of their size and monitored from the initial disordered state until coarsening. It is found that all components of cluster free energy vary linearly with the chemical potential of monomers, a property easy to implement into classical precipitation models.
\end{abstract}

Keywords: precipitation, simulation, clusters, Monte Carlo, free energy

\section{Introduction}

Whatever the complexity of precipitation in modern multi-components alloys, the fundamental laws describing the behaviour of spherical and coherent precipitates in a model binary alloy remain an essential reference. The description of this ideal case slightly improved over a century, mostly through theoretical advances. Forty years ago, the development of numerical tools based on Statistical Physics brought new tools to 
explore this classical subject. Then, the computing capabilities became sufficient to confirm the validity of classical theories, mostly thanks to Atomistic Kinematic Monte Carlo simulations (AKMC); at least for alloys of low concentration and low supersaturation [1].

In the mean time, aluminium industry, for instance, raised questionings about the validity of classical approaches for alloys of non negligible concentrations and/or supersaturations, typical situations which were out of reach of numerical simulations until twenty years ago.

To investigate this question, using $\mathrm{AKMC}$ simulations as a reference, $\mathrm{Al}_{3} \mathrm{Li}$ clusters embedded in an aluminium matrix were chosen as a model case, for both industrial and academical reasons (e.g. the high solubility of this phase).

The properties of clusters (including fluctuations and precipitates) were first explored with an oversimplified atomic model limited to second near-neighbour bonds [2]. To move beyond this necessary simplification, a classical atomic potential accounting for both first- and second- near neighbour bonds (noted NN1 and NN2, respectively) has been proposed recently [3]. Furthermore, a method to directly extract the free energy of clusters from AKMC simulations has been generalised and applied to AlLi alloys at the solubility limit, in the range $50^{\circ} \mathrm{C}-300^{\circ} \mathrm{C}$. In the present paper, this method is applied to clusters built during precipitation kinetics, in various conditions, in order to:

(i) check the validity of previous findings [2] using a more realistic potential,

(ii) try to express results in a form simple enough to be implemented into classical precipitation models like Cluster Dynamics (CD) [4] or KWN method [5].

It is reminded that in the Classical Nucleation Theory the free energy $F_{n}$ of clusters of size $n$ is based on the capillary approximation $F_{n}=G_{v}(n)+A(n) \gamma(n)$ where $G_{v}(n)$ is the nucleation energy, $\gamma$ the free surface energy and $A(n)$ the surface [6]. Note that this 
description fails to describe small clusters while the form validated by Perrini et al. [7] is not limited by cluster size but requires 4 parameters (cf. next section). By analogy with the capillary approximation, the first term is usually identified with a volume term while the other terms can be grouped in a generalised surface term depending on cluster size [6].

In classical mean field approximations (e.g. ideal or regular solid solution), by construction, $G_{v}$ varies during precipitation; if matrix frustration is taken into account, $\gamma$ is no longer constant [6]. In both cases these changes directly arise from the evolution of cluster distributions. By consequent, although to our knowledge it is never explicitly discussed in the literature, the evolution of cluster free energy during precipitation is not a new concept. The present work is a contribution to build a more general theory, not limited by solute concentration, supersaturation or cluster size.

\section{Methodology}

\subsection{A classical atomic model for AKMC simulations}

AKMC simulations (see [1] for details) of the precipitation of $\delta^{\prime} \mathrm{Al}_{3} \mathrm{Li}$ clusters $\left(\mathrm{L}_{2}\right.$ structure) in aluminium have been performed with an atomic model model based on $\mathrm{NN} 1$ and $\mathrm{NN} 2$ [3]. It is mostly derived from the parametrization proposed by Garland and Sanchez [8] for the two effective pair interaction parameters, i.e. $\omega_{2}=[1871 \mathrm{~J} / \mathrm{mol}]$ for $\mathrm{NN} 2$ and $\omega_{1}=-2 \omega_{2}$ for $\mathrm{NN} 1$. Although it is built to reproduce various thermodynamic and kinetic properties of this alloy, this atomic model should evolve, following the availability of DFT calculations. Nevertheless, it is very convenient for our present goal.

AKMC simulations were performed using a box of $200^{3}$ or $500^{3}$ atoms, depending on conditions and purposes. Figure 1 shows a typical sequence. 

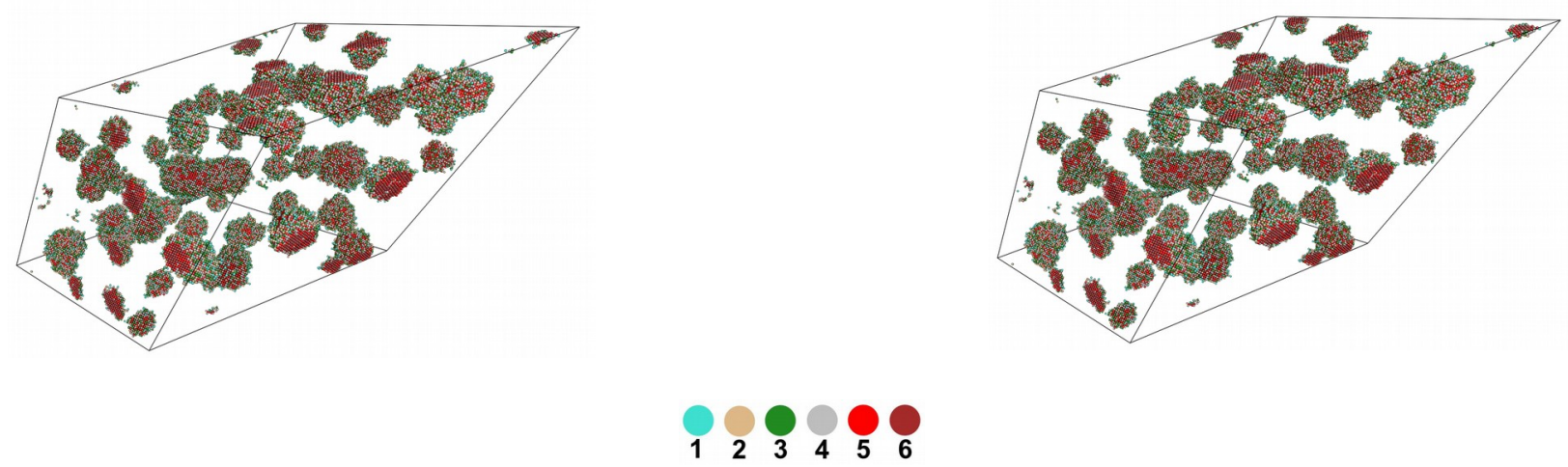

Figure 1: a typical sequence of precipitation. $T=85^{\circ} \mathrm{C}, \mathrm{C}_{0}=9 \%, 500^{3}$ sites. Only cluster of size larger than 100 (a) or 1000 (b) are shown. The colour indicates the number of solute neighbours. (a) early nucleation (b) coarsening

\subsection{Definitions}

The key quantity for precipitation is $F_{n}$, the free energy of clusters as a function of their size $n$ (the number of solute atoms). However, for modelling purposes, the required quantity is the difference of free energy $F_{n+1}-F_{n}$ which can be fit with a 4 coefficients function [7]:

$$
F_{n+1}-F_{n}=a+b\left((n+1)^{2 / 3}-n^{2 / 3}\right)+c\left((n+1)^{1 / 3}-n^{1 / 3}\right)+d \ln ((n+1) / n)
$$

To extract $a, b, c$ and $d$ from AKMC simulations, clusters are analysed following the 'CapRel' method based on the calculation of the so-called 'Capture' and 'Release' coefficients, $P_{n \rightarrow n+1}^{*}$ and $P_{n+1 \rightarrow n}^{*}$ respectively (see [3] for details). The ratio of these two coefficients is related to the difference of free energy in Equation (1):

$$
\frac{P_{n \rightarrow n+1}^{*}}{P_{n+1 \rightarrow n}^{*}}=\exp \left(\frac{h_{1}}{k_{B} T}\right) \exp \left(-\frac{F_{n+1}-F_{n}}{k_{B} T}\right)
$$

with $h_{1}$ the monomer enthalpy, $T$ the temperature and $k_{B}$ the Boltzmann constant. The symbol ' $*$ ' indicates that these coefficients account for the risk of coagulation between a cluster and its neighbours, when it captures a new solute atom. It has been shown that 
this ratio is the right quantity to be used in Cluster Dynamics (CD) to reproduce cluster distributions at equilibrium, which is an elementary test of validity [3].

Another fundamental quantity required to analyse results is the chemical potential of monomers $\mu_{1}[2]$ :

$$
\left(\frac{\mu_{1}}{k T}\right)=\left(\frac{h_{1}}{k_{B} T}\right)+\ln \left(\frac{C_{1}}{M_{1}}\right)
$$

with $C_{l}$ the concentration of monomers and $M_{1}$ the concentration of Al sites whose NN1 and NN2 are all occupied by Al atoms. In the following, $\bar{x}$ denotes the quantity $x$ normalised by $k_{B} T$; to simplify we note $\bar{a}^{*}=\bar{a}-\bar{h}_{1}$ and $\overline{\mu_{1}^{*}}=\bar{\mu}_{1}-\bar{h}_{1}$. At the solubility limit $a=\mu$, thus $\bar{a}^{*}=\ln \left(C_{1} / M_{1}\right) \quad$ [2-3]. Finally, with these notations, the quantity to fit writes:

$$
\frac{P_{n \rightarrow n+1}^{*}}{P_{n+1 \rightarrow n}^{*}}=\exp \left(-\left(\bar{a}^{*}+\bar{b}\left((n+1)^{2 / 3}-n^{2 / 3}\right)+\bar{c}\left((n+1)^{1 / 3}-n^{1 / 3}\right)+\bar{d} \ln ((n+1) / n)\right)\right)
$$

\section{Results}

This section examines the evolution of coefficients $\bar{a}^{*}, \bar{b}, \bar{c}$ and $\bar{d}$ during precipitation kinetics, analysing clusters with the 'CapRel' method [3].

\subsection{General behaviour}

Starting from a random distribution, a supersaturated system exhibits first a transient regime before reaching quasi-equilibrium (QE), as previously observed with a simplified atomic potential [2]. QE could be defined as a stage such that average properties of clusters are consistent with their distribution, like at true equilibrium [2]. Note that the onset of QE can be only assessed [2-3] but we checked that its choice is 
not critical for our purpose.

To compare different situations, it is convenient to report $P_{n \rightarrow n+1}^{*} / P_{n+1 \rightarrow n}^{*}$ versus the excess of chemical potential $\Delta \mu=\overline{\mu_{1}^{*}}(t)-\bar{\mu}_{1}^{*}(\infty)$, where $\overline{\mu_{1}^{*}}(\infty)$ is simply the value of $\overline{\mu_{1}^{*}}$ at the solubility limit (SL), supposed to be known [3].

In Figure 2, we have reported the measured values of $P_{n \rightarrow n+1}^{*} / P_{n+1 \rightarrow n}^{*}$ for several intermediate states between the beginning of QE ( $\Delta \mu=0.587)$ and the asymptotic state (SL) for $\mathrm{T}=200^{\circ} \mathrm{C}, \mathrm{C}_{0}=10 \%$. As can be seen in Figure 2, in these conditions, the change of cluster properties during precipitation is rather large, which is likely to be important for the nucleation and growth stages.

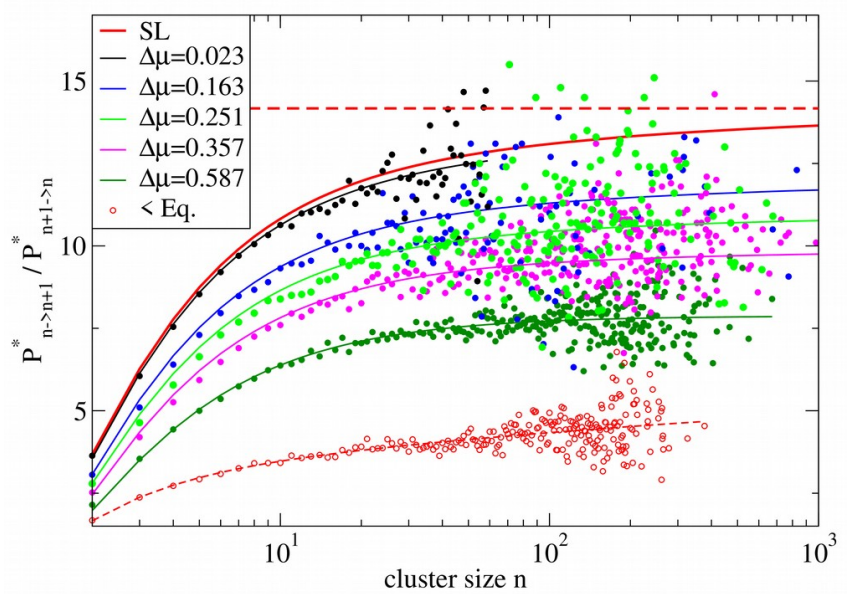

Figure 2: Evolution of $P_{n \rightarrow n+1}^{*} / P_{n+1 \rightarrow n}^{*}$ during precipitation for $\mathrm{T}=200^{\circ} \mathrm{C}$, $\mathrm{C}_{0}=10 \%$ (simulation box: $200^{3}$ sites). The upper bold curve "SL" refers to the solubility limit $\left(\mathrm{C}_{0}=6.3 \%\right)$ and the dashed line represents its asymptote. Symbols are related to measures performed on a given distribution of clusters obtained by AKMC simulations. The plain curves are the fits corresponding to the symbols of same colour. The lowest curve " $<$ Eq." is taken in the transient stage preceding QE. 
In Figure 3 we have reported the values of $\bar{a}^{\bar{*}}, \bar{b}, \bar{c}$ and $\bar{d}$ versus $\Delta \mu$ corresponding to the fits shown in Figure 2. Of course, there is no 'exact' solution for any of these fits but many approximative solutions. Thus, any justified simplification is welcome to secure the fitting procedure. For instance, at $200^{\circ} \mathrm{C} \bar{a}^{*} \sim \Delta \mu$, like at the solubility limit, thus to simplify the fit we imposed this value. Generally speaking, the challenge is not to find the best fit for each cluster distribution but to find the best compromise between accuracy and simplicity, convenient for the whole range of precipitation kinetics. It is worth noting that whatever the simplifications the consequences for the coarsening stage are negligible, because the $P_{n \rightarrow n+1}^{*} / P_{n+1 \rightarrow n}^{*}$ curve becomes necessarily very close to the asymptote (see Figure 2) which can be known with a good accuracy [3].

The lowest curve (open red symbols) in Figure 2 is an example of early state, taken before reaching QE, i.e. when Equation (2) is not yet fulfilled. As can be seen, this curve exhibits a very different shape, thus its fit would require a very different combination of parameters. Using such a state as starting point in Cluster Dynamics, for instance, to predict the evolution of cluster distributions would require a too complicated model. On the contrary, this task is fairly easy if the initial state is taken around the onset of QE, as shown in Figure 3: all components of $F_{n}$ vary linearly with $\Delta \mu$ 


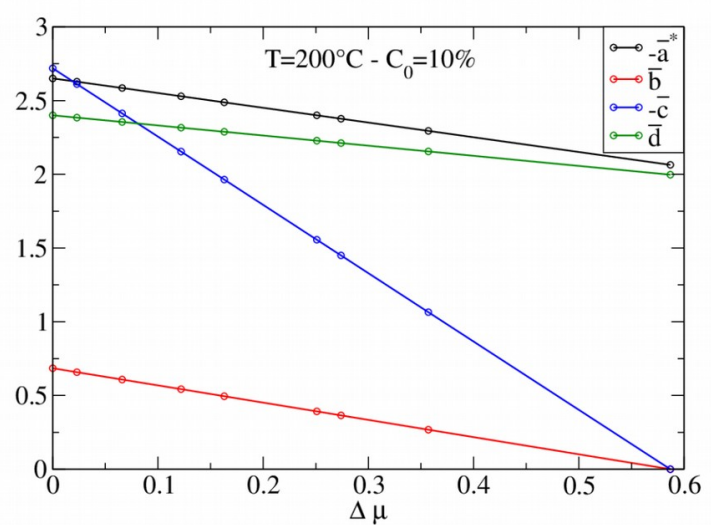

(a)

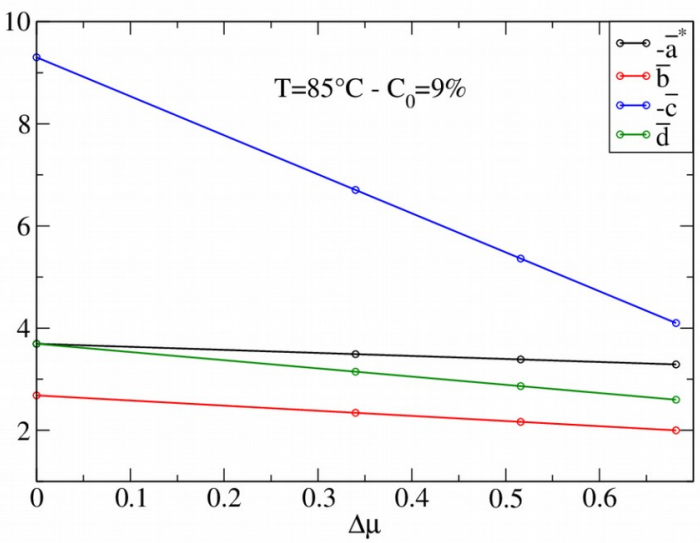

(b)

Figure 3: Evolution of the different coefficients used to fit $P_{n \rightarrow n+1}^{*} / P_{n+1 \rightarrow n}^{*}$ vs. $\Delta \mu$ during precipitation, in two very different cases of high concentration. The beginning of QE is at the right end of curves while the left one corresponds to the solubility limit.

This property of $P_{n \rightarrow n+1}^{*} / P_{n+1 \rightarrow n}^{*}$ vs. $\Delta \mu$ means that $F_{n}^{*}$ can be written as a combination of both initial (QE) and final (SL) solutions:

$F_{n}^{*}(\lambda)=\lambda F_{n}^{*}(Q E)+(1-\lambda) F_{n}^{*}(S L)$

where $\lambda$ is simply the ratio $\Delta \mu /(\mu(Q E)-\mu(S L))$, varying from 1 to 0 (at $t$ infinite).

\subsection{Influence of temperature}

Figure 2 is compared with two other situations of similar super-saturations: $\mathrm{T}=250^{\circ} \mathrm{C}$ and $\mathrm{C}_{0}=10.5 \%$ (Figure $4 \mathrm{a}$ ), $\mathrm{T}=85^{\circ} \mathrm{C}$ and $\mathrm{C}_{0}=4.75 \%$ (Figure $4 \mathrm{~b}$ ). Like in Figure 2 , the lower and upper curves correspond to the onset of QE and the solubility limit (SL), respectively. In addition, we have reported a few intermediate curves from nucleation to coarsening. For both cases we carefully fit the first curve, around the onset of QE, then 
we assumed that coefficients $\bar{a}^{*}, \bar{b}, \bar{c}$ and $\bar{d}$ vary linearly with $\Delta \mu$, as found in the previous case (Figure 2). All other fits shown in Figures $4 a-b$ follow this principle. As can be seen, these fits are in good agreement with measured data. The largest difference (a few \%) with the best solution is observed in Figure $4 \mathrm{a}$ in the range $n=5-30$. The analysis of cluster distributions suggests that this small discrepancy is associated with a percolating heterogeneous phase containing up to 300000 connected solute atoms (100000 for $\Delta \mu=0.264$ in Figure $4 \mathrm{~b}$ ) which coexists with a classical cluster distribution. This percolating phase appears during the nucleation stage and disappears before the coarsening stage, a behaviour which might be related to spinodal decomposition [8-9]. Although this behaviour might be interesting to explore, it is beyond the scope of the present work. However, it is interesting to note that even in such complex situations, the proposed procedure still provides good results for true clusters.

As can be seen in Figure 4a-b, although the temperature difference between these two cases is less than $200^{\circ} \mathrm{C}$, the curves exhibit very different shapes and take very different values. This behaviour makes extrapolation between two known temperatures rather difficult.

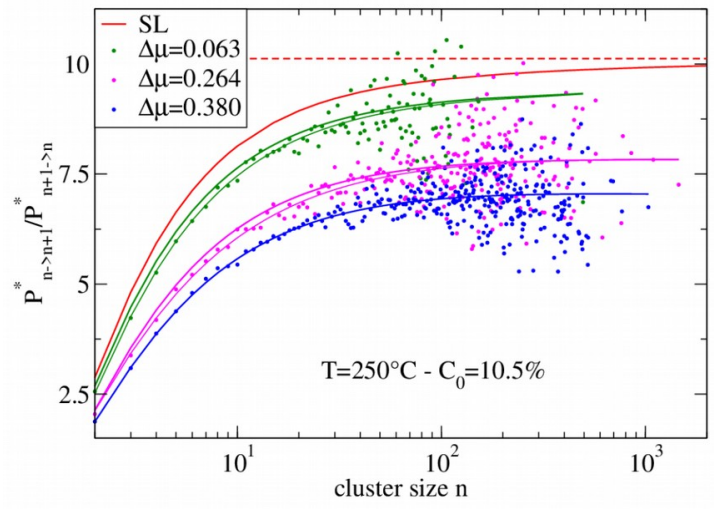

(a)

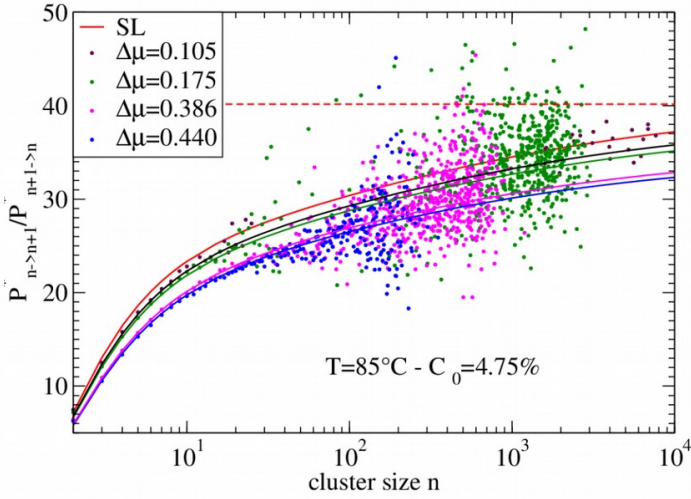

(b) 
Figure 4: Evolution of $P_{n \rightarrow n+1}^{*} / P_{n+1 \rightarrow n}^{*}$ during precipitation in two cases of comparable supersaturation $\left(\mathrm{C}_{0}-\mathrm{C}_{\mathrm{SL}}\right)$ (i.e. $2.64 \%$ in (a) and $2.05 \%$ in (b)). In both cases the lower curve is the best fit of AKMC data (symbols) for the beginning of QE, while the upper curve (SL) refers to the solubility limit. Thin lines in (a) are the best fits of AKMC data.

\subsection{Influence of concentration}

In Figure 5, for two values of $\Delta \mu$, taken at early nucleation and early coarsening, respectively, we reported $P_{n \rightarrow n+1}^{*} / P_{n+1 \rightarrow n}^{*}$ vs. $\Delta \mu$ at $\mathrm{T}=200^{\circ} \mathrm{C}$ for two close concentrations. As can be seen in Figure 5 the difference between two curves of same $\Delta \mu$ is small and the shape of curves is preserved. This suggests some relation between curves related to different concentrations, at constant temperature. Figure 5 shows also that this effect is obviously more important for nucleation and growth than for coarsening.

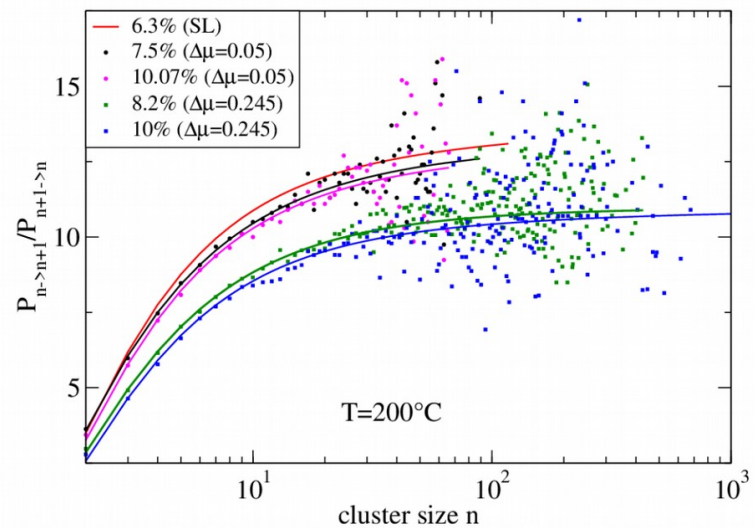


Figure 5: Influence of a small difference of solute concentration on $P_{n \rightarrow n+1}^{*} / P_{n+1 \rightarrow n}^{*}$. Symbols are related to AKMC simulations while plain curves of same colour are the corresponding fits. "SL" corresponds to the solubility limit.

Figure 6 compares two situations of very different concentrations at $\mathrm{T}=85^{\circ} \mathrm{C}$ : $\mathrm{C}_{0}=9 \%$, reported for three values of $\Delta \mu(0.682,0.516$ and 0.34$)$ and $\mathrm{C}_{0}=4.75 \%$ for $\Delta \mu=0.34$. First we fit the three first cases with the solution obtained for $\mathrm{C}_{0}=9 \%$ and reported in Figure 2b. Then to fit the fourth case with the same solution, we had to set $\Delta \mu \sim 0.34 / 1.5$. In other words, for a given temperature, the lower the initial excess of chemical potential $\Delta \mu$, the faster $F_{n}$ converges towards its asymptotic value. To confirm the generality of this property, all curves in figure $4 \mathrm{~b}$ for $\mathrm{C}_{0}=4.75 \%$ were fit with the data reported in Figure $3 b$ for $\mathrm{C}_{0}=9 \%$, using $\Delta \mu / 1.5$ instead of $\Delta \mu$ (the values at $\Delta \mu=0$ are unchanged by construction).

The quality of these fits proves that the relations between the different components of $P_{n \rightarrow n+1}^{*} / P_{n+1 \rightarrow n}^{*}$ are controlled only by temperature. Thus, to guess $P_{n \rightarrow n+1}^{*} / P_{n+1 \rightarrow n}^{*}$ for a concentration $C_{j}$, knowing the result at a concentration $C_{i}>C_{j}$, it is sufficient to determine the corresponding value of $\lambda\left(\mathrm{C}_{\mathrm{i}}\right)<1$ at the beginning of $\mathrm{QE}$ for the new case $\mathrm{C}_{\mathrm{j}}$

For this reason, it is preferable to spend computation time on a case of large supersaturation, e.g. $\mathrm{C}_{0}=9 \%$ here, which provides valuable information even with a $200^{3}$ simulation box. Then, to explore a smaller concentration, e.g. $\mathrm{C}_{0}=4.75 \%$ here, it is worth using a large simulation box to get the required information about clusters at the onset of QE, the earlier possible starting point for classical models. 


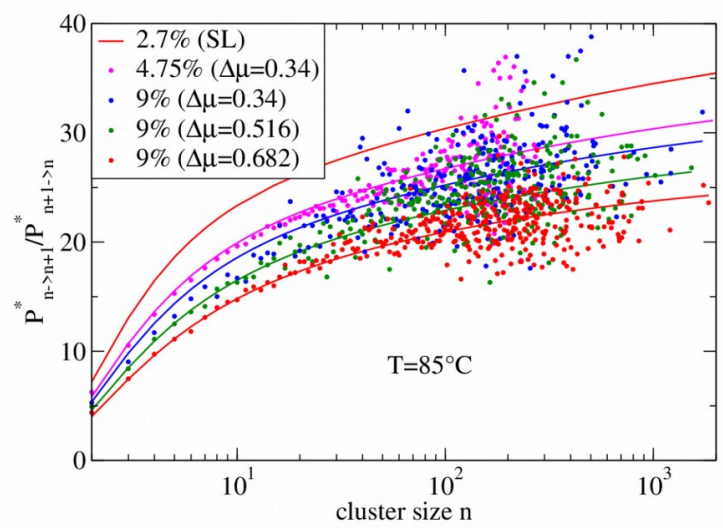

Figure 6: Influence of solute concentration on $P_{n \rightarrow n+1}^{*} / P_{n+1 \rightarrow n}^{*}$. Symbols are related to AKMC simulations while plain curves of same colour are the corresponding fits. "SL" corresponds to the solubility limit. Note the magenta curve related to a concentration half of others.

\subsection{General trends}

No general solutions for the variations of $\bar{a}^{*}, \bar{b}, \bar{c}$ and ${ }^{\bar{d}}$ with $\Delta \mu$ as functions of temperature can be provided because of the influence of concentration. Nevertheless some general trends emerge.

As already mentioned, for $\mathrm{T} \geqslant 200^{\circ} \mathrm{C}$ it is easy to impose $\bar{a}^{*}=\operatorname{Ln}\left(\mathrm{C}_{1} / \mathrm{M}_{1}\right)$ which means $\delta / \bar{a}^{*} \delta(\Delta \mu)=1$. On the contrary, at lower temperatures, at the onset of QE, $\bar{a}^{*}$ is already close to its asymptotic value, then $\delta / \bar{a}^{*} \delta(\Delta \mu)$ tends towards 0 . In other words, while $\bar{a}^{*}$ instantaneously adjust with $\Delta \mu$ at high temperature, $\bar{a}^{*}$ become less and less sensible to $\Delta \mu$ and concentration when T decreases. The evolution of $\delta \bar{b} / \delta(\Delta \mu)$ is more dependent on concentration, but its range of variation at a given temperature remains fairly small. The evolution of $\delta^{\bar{c}} / \delta(\Delta \mu)$ is roughly similar to that of $-\delta \bar{b} / \delta(\Delta \mu)$ with a 
much higher dependence on the concentration, which leads to large variations. Finally, $\delta^{\bar{d}} / \delta(\Delta \mu)$ is weakly sensible to the concentration and its values remain of the order of a few units.

\section{Conclusion}

In this paper, some previous findings about the evolution of cluster free energy during precipitation [2] have been confirmed with AKMC simulations, using a more classical potential. The 'CapRel' method [3] has been used to extract the free energy of clusters during precipitation kinetics.

For the first time it is shown that the evolution of cluster free energy $F_{n}$ and its components are linear functions of $\Delta \mu$, the excess of chemical potential of monomers. The simplicity of this result makes it easy to implement into classical precipitation models. Ideally, $\Delta \mu$ should be managed simultaneously in a consistent way; it depends on $\mathrm{M}_{1}$ (Equation (3)), a variable closely related to the notion of cluster volume (discussed in a next paper). It is also shown how the information required to upgrade classical models can be obtained while minimizing the amount of atomistic calculations. 


\section{Acknowledgements}

Dr. E. Clouet is gratefully acknowledged for providing his AKMC package. Dr C. Sigli is warmly acknowledged for numerous fruitful discussions.

This research did not receive any specific grant from funding agencies in the public, commercial, or not-for-profit sectors.

\section{References}

[1] F. Soisson and G. Martin, Monte Carlo simulations of the decomposition of metastable solid solutions: Transient and steady-state nucleation kinetics, Phys. Rev. B 62(1) (2000) pp. 203-214.

[2] J. Lépinoux and C. Sigli, Multiscale modelling of precipitation in concentrated alloys: from atomistic Monte Carlo simulations to cluster dynamics: I thermodynamics, Phil. Mag. 98(1) (2018) pp. 1-19.

[3] J. Lépinoux and C. Sigli, Extracting free energy of clusters in concentrated binary alloys from atomistic Monte Carlo simulations, Modelling. Simul. Mater. Sci. Eng. 27 (2019) p. 085001

[4] E. Clouet, A. Barbu, L. Laé and G. Martin, Precipitation kinetics of $\mathrm{Al}_{3} \mathrm{Zr}$ and $\mathrm{Al}_{3} \mathrm{Sc}$ in aluminum alloys modeled with cluster dynamics, Acta Mater. 53 (2005) pp. 23132325

[5] M. Perez, M. Dumont and D. Acevedo-Reyes, Implementation of classical nucleation and growth theories for precipitation, Acta Mater. 56 (2008) pp.2119-2132.

[6] E. Clouet, Modeling of nucleation processes, in Fundamentals of Modelling for Metals Processing, D.U. Furrer and S.L. Semiatin (Eds.), Materials Park, OH, ASM 
Handbook, vol. 22A Fundamentals of modelling for metals processing (2010) pp.203219.

[7] A. Perini, G. Jacucci and G. Martin, Cluster free energy in the simple-cubic Ising model, Phys. Rev. B 29 (1984) pp. 2689-97

[8] J.S. Garland and J.M. Sanchez, Cluster variation method calculation of the metastable aluminium-lithium phase diagram, in Kinetics of Ordering Transformations in Metals, H. Chen and V.K. Vasudevan (Eds.), TMS, Warrendale, PA, (1992) pp. 207216.

[9] Y.H. Wei and S.T. Wang, Experimental evidence for spinodal decomposition along with simultaneous ordering in Al-12.7 at\% Li alloy, Materials Letters 28 (1996) pp.123127 